\title{
Electron Heating in a Relativistic, Weibel-Unstable Plasma
}

\author{
Rahul Kumar* \\ Physics Department, Ben-Gurion University, Be'er-Sheba 84105, Israel E-mail: \\ rahulittegmail.com
}

The dynamics of two initially unmagnetized relativistic counter-streaming homogeneous ionelectron plasma beams are simulated in two dimensions using the particle-in-cell (PIC) method. It is shown that current filaments, which form due to the Weibel instability, develop a large scale longitudinal electric field in the direction opposite to the current carried by the filaments as predicted by theory. This field, which is partially inductive and partially electrostatic, is identified as the main source of net electron acceleration, greatly exceeding that due to magnetic field decay at later stages. The transverse electric field, though larger than the longitudinal one, is shown to play a smaller role in heating electrons, contrary to previous claims.

The 34th International Cosmic Ray Conference,

30 July- 6 August, 2015

The Hague, The Netherlands

${ }^{*}$ Speaker. 


\section{Introduction}

Collisionless shocks forming in astrophysical environments are believed to be mediated by electromagnetic instabilities in which ions are scattered by the resulting magnetic field fluctuations. In particlular, the Weibel instability [1], which causes fast growth of strong magnetic field at smallscale in anisotropic plasma flow, has received much attention as the main isotropization mechanism that leads to shock transition in free-streaming ejecta from violent astrophysical events.

Numerical simulations suggest presence of large scale electric field in and around the current filaments forming just ahead of the shock and ions are found to decelerate due to this electric field. The same electric field that decelerates ions should also accelerate electrons. Details of the acceleration mechanism has been discussed in [2].

In order to understand the heating of electrons in foreshock region of relativistic shocks we simulate, using the kinetic PIC method, development of the Weibel instability in two relativistic counter-streaming plasma beams, which resembles the precursor of a relativistic shock, but simpler and more idealized and therefore more suitable for resolving certain fundamental questions. The beams are taken to be homogeneous and fully interpenetrating at the beginning, so the time development of the instability in our simulation imitates the spatial development of the instability in the foreshock regions, where later times in our simulation correspond the regions closer to the shock fronts. Our quantitative analysis suggests that the most of the heating of upstream electrons is due to the longitudinal electric field. The transverse electric field, though much stronger than the longitudinal, has negligible effect on net acceleration of electrons.

\section{Numerical Simulation}

We use an electromagnetic PIC method to simulate relativistic counter-streaming beams of ions and electrons in two dimensions. The simulation is initialized by placing ion-electron pairs at uniformly chosen random locations in a two dimensional square box in x-y plane. Initially ion and electron in each pair are moving in opposite directions along the $\mathrm{x}$-axis with Lorentz factor $\gamma_{0}$. Half of the total number of pairs have ions moving along the positive $\mathrm{x}$-axis while an equal number of another half pairs have ions moving along the negative x-direction, hence creating two oppositely streaming neutral plasma beams of equal intensity, where each beam has a net current. The initial condition ensures that the simulation is initially charge and current free and that Maxwell equations are satisfied over scales where there are equal numbers of forward and backward moving electronion pairs, but at the grid scale, local fluctuations in the number of forward and of backward movers imply a net small scale current. This noise level, however, is reddened by smoothening the grid scale current. As the electrons and ions in the pairs separate from each other, the electric and magnetic field grow from the noise in the current generated by streaming electrons and ions. Since we simulate an initially unmagnetized plasma, only out-of-plane magnetic field $B_{z}$ and in-plane electric fields $E_{x}$ and $E_{y}$ are excited as the Weibel instability set in. We impose periodic boundary condition in both $\mathrm{x}$ and $\mathrm{y}$ directions for both particles and fields.

Here we discuss results mainly from the two largest simulations we have attempted in terms of physical size and evolution time. Ion to electron mass ratio $m_{i}: m_{e}$ for the two reported simulations are 16:1 and 64:1, and are henceforth referred to as $M_{16}$ and $M_{64}$, respectively. All the figures in 

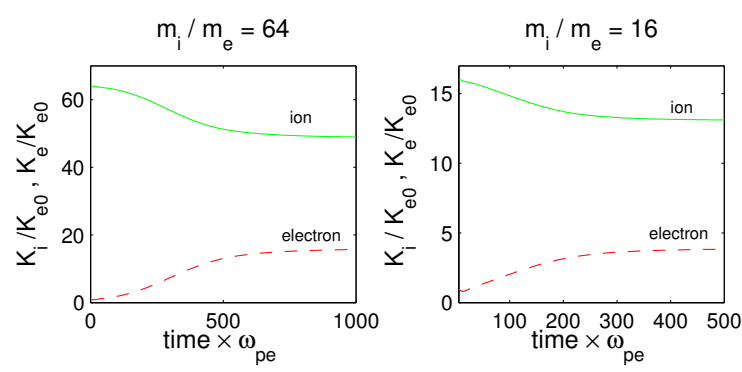

Figure 1: Evolution of mean kinetic energy of electrons $K_{e}$ (dashed red ) and ions $K_{i}$ (solid green) are shown for two different ion to electron mass ratios (normalized to the initial kinetic energy of electrons $K_{e 0}$ ) . Electrons heating due to the Lenz electric field is significant until the ion current filaments are disrupted. By the end of the simulation electrons are heated to about $1 / 3$ of the ions kinetic energy in both cases consider here.

this paper are for ion to electron mass ratio of 64:1, i.e. simulation $M_{64}$, unless otherwise stated. The physical sizes of the box for $M_{16}$ and $M_{64}$ are $500 \mathrm{c} / \omega_{p e} \times 500 \mathrm{c} / \omega_{p e}$ and $1000 \mathrm{c} / \omega_{p e} \times 1000$ $\mathrm{c} / \omega_{p e}$, respectively, where $\mathrm{c}$ is the speed of light in vacuum and $\omega_{p e}=\sqrt{4 \pi n_{0} e^{2} / m_{e} \gamma_{0}}$ is the initial electron plasma frequency, where $e$ is the charge of an electron and $n_{0}$ is the initial number density of electrons, or by charge neutrality, of ions. Both simulations were resolved to $1 / 10$ th of the initial electron skin-depths. Initially there are 32(8) particles per unit cell of the simulation box for $M_{16}\left(M_{64}\right)$ and the simulation was evolved for 500 (1000) plasma time $\omega_{p e}^{-1}$. Initially the ions and electrons are moving along the $\mathrm{x}$-axis with initial Lorentz factor $\gamma_{0}=10$ in the both cases.

\subsection{Net work done by the electric field}

In figure 1 we show the time evolution of the total kinetic energy of electrons and ions. It shows that most of the energy exchange between ions and electrons takes place during the filamentation stage of the instability, when magnetic field is still growing in strength. We have run the simulation for long enough to capture most of the heating phase and long after the rapid heating phase ends, electrons are found to have acquired a substantial part of the ions's kinetic energy, in agreement with the estimate presented above. Even long after the breaking of filaments, ions are not completely thermalized and continue to lose energy and the heating of electrons continues, though at a much lower rate.

While the magnetic field scatter the charged particles by altering their trajectories, the only force that changes energy of the plasma particles is due to the electric field in the lab frame. From our PIC simulations we separate out work done by the two orthogonal components of the electric field, namely $E_{x}$ and $E_{y}$, on electrons as well as on protons. As evident from Figure 2, in both the simulations reported here the change in total kinetic energy of electrons and protons is apparent to be mainly due to the longitudinal electric field $E_{x}$. The net work done by the transverse electric field $E_{y}$, which is rather stronger than the longitudinal electric field, is vanishingly small.

\section{References}

[1] Weibel E. S., 1959, Phys. Rev. Lett., 2,3

[2] Kumar R., Eichler D., Gedalin M., 2015, The Astrophysical Journal, 806,165 

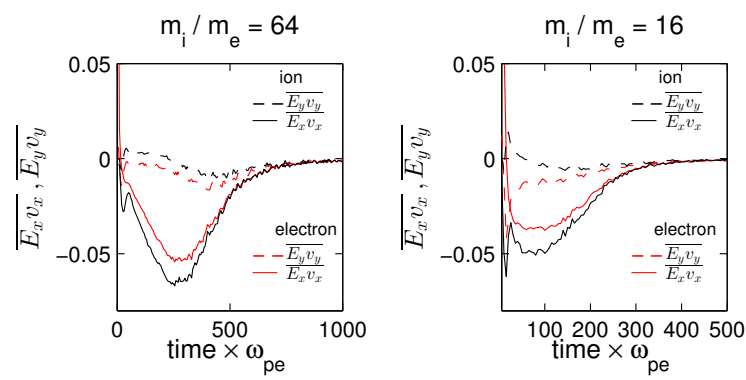

Figure 2: The rate of energy gained (lost) by electrons (protons), as a function of time, due to $E_{x}$ and $E_{y}$, i.e. $\overline{E_{x} v_{x}}$ and $\overline{E_{y} v_{y}}$, respectively (in units of $c^{2} \sqrt{4 \pi \gamma_{0} m_{e}}$ ), are shown by solid and dashed red (black) curves, respectively. The quantities $\overline{E_{x} v_{x}}$ and $\overline{E_{y} v_{y}}$ are the mean values of $E_{x} v_{x}$ and $E_{y} v_{y}$, respectively, computed for the sample of particles (few percent of the total number of particles) which were initially homogeneously distributed over the simulation box. The left and right panels are for ion to electron mass ratios 16:1 and 64:1, respectively. Though the transverse electric field $E_{y}$ is much larger in magnitude than the longitudinal electric field $E_{x}$, its contribution to the electron acceleration and ion deceleration (dashed lines) is negligible as comparable to the heating due to $E_{x}$. 